\title{
Erratum to: Semigroup identities in the monoid of triangular tropical matrices
}

\section{Zur Izhakian' ${ }^{1}$}

Received: 3 March 2016 / Accepted: 3 March 2016 / Published online: 4 April 2016

(C) Springer Science+Business Media New York 2016

\section{Erratum to: Semigroup Forum (2014) 88:145-161 DOI 10.1007/s00233-013-9507-6}

One of the conditions in the main result of the paper [1] is too strict as it stands: Theorem 4.8 should be replaced as follows.

Theorem 4.8 Any two triangular tropical matrices $X, Y \in U_{n}(\mathbb{T})$ having the same diagonal satisfy the (nontrivial) identities:

$$
\widetilde{w}_{(C, P, n-1)} X \widetilde{w}_{(C, P, n-1)}=\widetilde{w}_{(C, P, n-1)} Y \widetilde{w}_{(C, P, n-1)},
$$

where $\widetilde{w}_{(C, P, n-1)}$ is any word having as its factors all the words of length $n-1$ generated by $C=\{X, Y\}$ of powers $P=\{1,2, \ldots, n-1\}$, such that $\widetilde{w}_{(C, P, n-1)} X \widetilde{w}_{(C, P, n-1)}$ and $\widetilde{w}_{(C, P, n-1)} Y \widetilde{w}_{(C, P, n-1)}$ are generated by $C$ and powers $P$.

The proof of the theorem appears in [2, Theorem 4.5].

\section{References}

1. Izhakian, Z.: Semigroup identities in the monoid of triangular tropical matrices. Semigroup Forum 88(1), 145-161 (2014)

2. Izhakian, Z.: Semigroup identities of tropical matrix semigroups of maximal rank. Semigroup Forum (to appear). http://link.springer.com/article/10.1007\%2Fs00233-015-9765-6

The online version of the original article can be found under doi:10.1007/s00233-013-9507-6.

$凶$ Zur Izhakian

zzur@post.tau.ac.il; zzur@math.biu.ac.il

1 Department of Mathematics, University of Bremen, 28359 Bremen, Germany 